\title{
Male panda (Ailuropoda melanoleuca) urine contains kinship information
}

\author{
LIU DingZhen ${ }^{1 \dagger}$, WEI RongPing ${ }^{2}$, ZHANG GuiQuan², YUAN Hong ${ }^{1}$, WANG ZhiPeng ${ }^{1}$, SUN Lixing ${ }^{3 \dagger}$, \\ ZHANG JianXu ${ }^{4} \&$ ZHANG HeMin ${ }^{3}$ \\ ${ }^{1}$ Key Laboratory for Biodiversity Science and Ecological Engineering of Ministry of Education China \& Institute of Ecology, Beijing \\ Normal University, Beijing 100875, China; \\ ${ }^{2}$ China Research and Conservation Center for the Giant Panda, Wolong 623006, China; \\ ${ }^{3}$ Department of Biological Science, Central Washington University, WA 98926-7537, USA; \\ ${ }^{4}$ State Key Laboratory of Integrated Management of Pest Insects and Rodents in Agriculture, Institute of Zoology, Chinese Academy \\ of Science, Beijing 100080, China
}

Chemical communication plays an important role in kin selection and mate choice in mammals. The covariance of odor-genes of rodents has been documented and kinship odor has been proposed and termed, yet little is known of the relationship between genetic relatedness and chemical composition of kinship odors. Giant pandas (Ailuropoda melanoleuca) rely substantially on chemical communication to mediate their social interactions. To examine the relationship between genetic relatedness and compounds in the urine/anogenital gland secretions, we compared the similarities between genetic relatedness and the chemical profiles of anogenital gland secretions and urine via lineage construction and GC-MS (gas chromatography and mass spectrometry). We found that information about kinship odors was present only in the urine of male adults in the mating season but absent in the non-mating season. Adult females and all sub-adults did not have such kinship odors in either mating or non-mating season. Therefore, kinship odor in the panda was contingent on age, sex, and season. This is the first report about the condition-dependent expression of kinship odor, which may have a significant implication in the practice of panda conservation in relation to chemical communication and sexual selection.

kinship odor, giant panda, Ailuropoda melanoleuca, anogenital gland secretions, urine

Most mammals use chemical signals to communicate a wide range of information in their social interactions. Chemical communication is particularly important in rodents, carnivores, and ungulates, which have highly sensitive olfactory systems ${ }^{[1]}$. The coevolutionary process between signalers and receivers drives chemical signals to become diverse and specific. Many mammals have evolved several glandular and non-glandular sources to communicate different types of information $^{[2,3]}$. Carnivores, in particular, use chemical communication substantially and anal (or anogenital) gland is present in most carnivores ${ }^{[4]}$, although it is still unclear whether it is due to convergence or divergence evolution. The gland secretion itself and its contaminating feces are rich sources of chemical signals. In addition, urine also widely contains chemical information in carnivores as well as other mammals ${ }^{[5,6]}$.

Odors that serve to distinguish one individual from another member of the species are determined by polymorphic genes ${ }^{[7]}$. Genetically related individuals show similar odors, and kinship odor was first demonstrated to be present in golden hamsters (Mesocricetus auratus) ${ }^{[8]}$ and wild beavers (Castor canadensis) ${ }^{[9]}$. Similar results

\footnotetext{
Received November 16, 2007; accepted March 20, 2008 doi: $10.1007 / \mathrm{s} 11434-008-0373-7$

†Corresponding author (email: dzliu@bnu.edu.cn, lixing@cwu.edu)

Supported by the National Natural Science Foundation of China (Grant Nos. 30470233, 30170169 and 30230080), Research Fund for the Doctoral Program of Higher Education (Grant No. 98002717), and China Wildlife Conservation Association (Grant Nos. 96035 and WH0309)
} 
were later found on Turkish hamsters (Mesocricetus brandti $)^{[10]}$ and giant mole rats (Cryptomys mechowi $)^{[11]}$. Behavioral tests also prove that Belding's ground squirrels (Spermophilus beldingi) can use odors to make accurate discriminations among unfamiliar kin, and a closely related species (S. lateralis) similarly produces kin labels and discriminates among kin $^{[12]}$. Although the exact genetic mechanism for the production of kinship odor is yet to be elucidated, several innovative studies have demonstrated that genes and chemical secretions covary ${ }^{[13,14]}$. The association between odor and genes thus was termed as "odor-genes covariance" by Todrank and $\mathrm{Heth}^{[15]}$. These findings indicate that kinship odors may be prevalent in mammals. Despite the fact that few chemical analyses are currently available about the chemical nature and composition of kinship odors, kinship odors might contain multiple constituents and possibly belong to the volatile and/or semivolatile components in the odor based on information uncovered from the beaver ${ }^{[9]}$ and the golden hamster ${ }^{[8]}$.

Despite the scarcity of genetic information, the two behavioral functions of kinship odors are relatively well understood. One is for maintaining an optimal breeding system, and the other for mediating altruistic behavior ${ }^{[16]}$. Which function is more important, however, depends on social contingencies. In gregarious animals, both functions may be important. In solitary animals, kinship odors may be critical in finding a mate with appropriate genetic relatedness, but its role in mediating altruistic interactions may be minimal. In any case, fine kin recognition is often the key prerequisite for excising either of the two functions ${ }^{[17,18]}$.

The giant panda (Ailuropoda melanoleuca) is a solitary animal inhabiting fragmental and mountainous areas of Sichuan, Shaanxi and Gansu provinces, China. Relatively poor in eyesight and hearing ability, pandas substantially use anogenital gland secretion (AGS) and urine to regulate their social behaviors, such as territorial advertisement, mate seeking, assessing female's reproductive status ${ }^{[19-21]}$. Previous behavioral and chemical assays suggest that these two sources of scent marks contain information about individual, sex, and age ${ }^{[22-26]}$. Both wild and captive observations reveal that pandas exhibit mate choice ${ }^{[27,28]}$. Without males' participating in providing parental care for the offspring, females in a polygamous system are much more choosier on mates than those in a monogamous system ${ }^{[29,30]}$. This may be particularly true for mono-estrous female pandas because they are receptive for only $1-3 \mathrm{~d}$ in the mating season (spring estrus: from late February to early June each year). If they are not conceived within this narrow time window, they will miss a year of reproduction, despite rare occasions of fall estrus ${ }^{[31]}$. With an average longevity of 13 years and reproductive life of 5 (for males) or 6 (for females) years in the wild after 1 year old $^{[32]}$, loss of one breeding season is a substantial reduction $(16 \%-20 \%)$ in their lifetime reproductive success $^{[28]}$. With such a large cost at stake, female pandas should choose best mates that can maximize their reproductive success. This, of course, includes genetic compatibility with an optimal relatedness between the mates.

Based on the above-mentioned reasoning, we speculate that kinship odor should be present in the panda because of its vital roles for especially females. Previous evidence has shown that pandas predominately use scent marks in their social life ${ }^{[19,20]}$, AGS and/or urine thus are hypothesized to be the possible source(s) of kinship odor for kin discrimination ${ }^{[17,18,33,34]}$. By following this line, the hypothesized kinship odor, if it does exist, would lead us to predict that closely related individuals share more common features than distantly related individuals in the chemical profile of the secretion sources (AGS and/or urine). We tested this prediction in this study by relating the similarity in the chemical profile of urine and AGS to the genetic relatedness between pandas.

\section{Materials and methods}

\subsection{Animals and sample collections}

The AGS samples for this study were collected from 24 captive pandas at the mating season of 2000 and 2001. The urine samples were collected from 13 pandas at the mating season of 2002 and 31 pandas at the non-mating season of 2001, 2002 and 2004 at the China Research and Conservation Center for the Giant Panda at Wolong, Sichuan, China (Table 1). We first classified the subjects into four sex-age groups (adult males, adult females, sub-adult males, and sub-adult females) based on the criteria by Wang and $\mathrm{Hu}^{[35]}$. The details about animal management and sample collection (AGS and urine) are available elsewhere ${ }^{[24,36]}$.

In short, we collected AGS samples with clean tampons directly rubbing on the anogenital glands of live pandas sedated for collecting semen or artificial insemi- 
Table 1 Number of subjects from which samples were collected in the mating and non-mating seasons

\begin{tabular}{lcccccc}
\hline \multirow{2}{*}{ Subjects } & \multicolumn{2}{c}{ Mating season } & & \multicolumn{2}{c}{ Non-mating season } \\
\cline { 2 - 3 } \cline { 5 - 6 } & AGS & urine & & AGS & urine \\
\hline Adult males & 5 & $6\left(4^{\mathrm{a}}\right)$ & & - & $14\left(1^{\mathrm{b})}\right)$ \\
Adult females & 6 & $7\left(3^{\mathrm{a})}\right)$ & & - & 10 \\
Sub-adult males & 6 & - & & - & 6 \\
Sub-adult females & 7 & - & & - & 1 \\
Total individuals & 24 & 13 & & - & 31
\end{tabular}

a) Numbers of repeatedly used animals in the non-mating season for urine samples collection; b) numbers of repeatedly collected samples in 2001 and 2004.

nation. Some samples were also collected from new, clean scent marks on walls, cement surfaces, or stones during 2001. Secretions of each subject were collected once, but we kept two glass vials for each sample. We immediately put samples in clean vials, and sealed and stored them at $-20^{\circ} \mathrm{C}$ without any chemical treatment. For the urine samples, we collected samples with clean syringes from the cement floor between 9:00 and 14:00 h. At least two bottles were used for holding each urine sample $(5-10 \mathrm{~mL})$ from one individual. Both AGS and urine samples collection in each season lasted less than 2 weeks. Samples were immediately transferred into clean vials with lids lined with Teflon and stored at $-20^{\circ} \mathrm{C}$. Both the AGS and urine samples were packed with ice and air-shipped to the laboratory in Beijing Normal University (about 2-h flight) and stored at $-20^{\circ} \mathrm{C}$ until analysis. We could not collect AGS samples at the non-mating season during the 3-year study period because of the strict management rules for especially adult female pandas during pregnancy and breeding. Another reason is that in the non-mating season, sub-adults were mostly mixed and raised in one cage, and adult males were released into semi-natural enclosures not accessible for us to collect scent marks. For similar reasons, we only succeeded in obtaining adult male and female urine samples, sub-adult male urine samples, and one sub-adult female urine sample (excluded in our analysis due to the small sample size). For the collection of urine samples from sub-adults mixed in one cage/enclosure in the non-mating season, the animals were monitored continuously from 9:00 to 14:00 h by the zoo keepers. As urinating was observed in a particular animal, the zoo keeper would rapidly enter the cage to collect urine samples, and the sample site on the cement floor was washed with water followed by cleaning with a piece of dry swabbing cloth.

\subsection{GC-MS analysis}

We used GC-MS (gas chromatography combined with mass spectrometry) to obtain information about the chemical compounds and their abundances for the two types of secretion from each individual. We tentatively identified compounds by searching for the library of the National Institute of Standards and Technology (NIST) and characterized each component by comparing it with the mass spectra in the library and literatures ${ }^{[26]}$. Details about the GC-MS analysis and the compounds from AGS and urine are as described by our previous papers ${ }^{[24,36]}$. The GC-MS analyses for the urine and AGS samples collected in 2000 through 2002 were conducted in November of 2002, and the urine samples collected in 2004 were analyzed in November and December of 2004.

\subsection{Data analysis}

Since both secretions contain chemical compounds usable for coding information about sex, age, and reproductive status ${ }^{[24,36]}$, they are logically unlikely to be used for coding information about genetic relationship, for they lack individual variation ${ }^{[2,9]}$. We thus excluded these compounds from further analysis. After this, we converted the amount of each remaining compound into relative amount based on its proportion in the total amount of all the compounds combined ${ }^{[9]}$.

To quantify the difference in the multi-component urine or AGS between two individuals, we used the Euclidean distance as the dissimilarity index for the chemical profile of the secretions. Meanwhile, we calculated the pair-wise genetic relatedness by using the software LINEAGE (Trial version, Cornell University) based on the breeding histories of our subjects available in the studbook ${ }^{[37]}$. Finally, we related the similarity in chemical profile to that in genetic relatedness using Spearman's rank correlation coefficient. All analyses were performed in SPSS 10.0 with the level of significance set at $\alpha=0.05$.

\section{Results}

Twenty eight compounds left in AGS after removing those compounds show effects of age and sex (Table 2). Most of those compounds are fourteen or more carbochain compounds with molecular weights of more than 200. They are 5 aldehydes, 7 fatty acid esters, 4 steroids and 1 acid amide. For the urinary compounds, however, 
Table 2 Tentatively identified compounds in the anogenital gland secretion excluding the compounds of showing effects of sex and age differences

\begin{tabular}{|c|c|c|c|c|}
\hline Peak No. & $\mathrm{RT}(\mathrm{min})$ & Tentatively identified compounds ${ }^{\text {a) }}$ & Molecular weight & Classification \\
\hline 4 & 18.54 & tetradecanal & 212 & aldehyde \\
\hline 8 & 19.78 & tetradecanoic acid, ethyl ester & 256 & fatty acid ester \\
\hline 10 & 20.24 & hexadecanal & 240 & aldehyde \\
\hline 13 & 21.47 & pentadecanoic acid, ethyl ester & 270 & fatty acid ester \\
\hline 15 & 21.95 & octadecanal & 268 & aldehyde \\
\hline 16 & 22.63 & $n$-hexadecanoic acid & 256 & saturated fatty acid \\
\hline 19 & 23.69 & eicosanal & 296 & aldehyde \\
\hline 22 & 24.90 & heptadecanoic acid, ethyl ester & 298 & fatty acid ester \\
\hline 23 & 25.43 & docosanal & 240 & aldehyde \\
\hline 24 & 25.57 & Z9,Z12-octadecadienoic acid & 280 & unsaturated fatty acid \\
\hline 27 & 26.59 & octadecanoic acid, ethyl ester & 312 & fatty acid ester \\
\hline 29 & 28.22 & 9-methyl-octadecanoic acid, ethyl ester & 326 & fatty acid ester \\
\hline 30 & 29.25 & Z9-octadecenamide & 281 & acid amide \\
\hline 32 & 29.75 & eicosanoic acid, ethyl ester & 340 & fatty acid ester \\
\hline 33 & 31.16 & pentacosane & 352 & alkane \\
\hline 34 & 32.35 & hexacosane & 366 & alkane \\
\hline 35 & 33.44 & heptacosane & 380 & alkane \\
\hline 36 & 34.13 & a steroid & $?$ & steroid \\
\hline 37 & 34.44 & a steroid & $?$ & steroid \\
\hline 38 & 34.57 & squalene & 410 & terpene \\
\hline 41 & 35.15 & cholesteryl benzoate & 490 & steroid \\
\hline 42 & 35.30 & $3 \beta$-cholesta-4,6-dien-3-ol & 384 & steroid \\
\hline 43 & 35.56 & cholesta-3,5-diene & 368 & steroid \\
\hline 47 & 37.80 & a terpene & $?$ & terpene \\
\hline 49 & 38.21 & cholesterol & 386 & steroid \\
\hline 54 & 39.35 & cholesta-3,5-dien-7-one & 382 & steroid \\
\hline 55 & 39.73 & campesterol & 400 & steroid \\
\hline 56 & 39.91 & cholest-4-en-3-one & 384 & steroid \\
\hline
\end{tabular}

a) Compounds are numbered in the order of increasing GC retention time. Trace components are not listed. "?", Represents the compound that could not be identified.

most of them are compounds with a medium retention time. They are 5 alcohols, 3 aldehydes, 8 alkanes, 2 acid amides, 8 ethers, 3 fatty acid esters, 5 ketones, 9 phenols and 2 saturated fatty acids (Table 3 ).

For the relation between odor and genetic relatedness, we found no significant correlation in similarity between the chemical profile of urine and genetic relatedness for adult males, adult females and sub-adult males in the non-mating season. Nor did we find any significant correlation in similarity between urine or AGS and genetic relatedness for adult females, sub-adult males, or subadult females in the mating season (see Table 4 for the detailed information about all the statistical results). The only significant correlation was the similarity between urinary chemical profile and genetic relatedness in adult males in the mating season $\left(r_{s}=0.851, n=15, P<0.001\right)$.

\section{Discussion}

Despite incompleteness in our samples, especially those from AGS in the non-mating season (Table 1), due to strict regulations for panda care and welfare that hindered our sample collection especially for $\mathrm{AGS}^{[24]}$, our results are still revealing. The positive correlation in similarity between genetic relatedness and chemical profile in adult males in the mating season stood out to be highly significant. This supported the prediction based on our hypothesis that kinship odor exists in the giant panda. Here, for the first time, we report that kinship odor may be present in a large, non-rodent mammalian species. Our results were, however, intriguing in several ways: the kinship odor as we found depended on sex, age, and season.

First, why did males, not females, have kinship information in the urine? Although both males and females exhibit mate preference ${ }^{[27]}$, observations show that males normally engage in fights with other males for mating with females in the wild ${ }^{[19,28]}$. Females have been observed to demonstrate mate choice and may mate with more than one males in a mating season ${ }^{[28]}$. Thus, the panda falls in the general pattern of sexual selection in most mammals where males compete and females 
Table 3 Tentatively identified compounds in the urine of the giant panda excluding the compounds showing effects of age, sex and season

\begin{tabular}{|c|c|c|c|c|}
\hline Peak No. & $\mathrm{RT}(\min )$ & Tentatively identified compounds ${ }^{\text {a) }}$ & Molecular weight & classification \\
\hline 1 & 11.36 & 1-(1-methylethoxy)-butane & 116 & alkane \\
\hline 2 & 11.51 & 3-hydroxy-butanoic acid, ethyl ester & 132 & fatty acid ester \\
\hline 3 & 11.80 & 3,6-dimethyl-4,5-octanediol, & 174 & alcohol \\
\hline 7 & 12.86 & butane, 2-ethoxy-butane & 102 & alkane \\
\hline 8 & 13.11 & an ether & $?$ & ether \\
\hline 9 & 13.17 & an ether & $?$ & ether \\
\hline 10 & 13.21 & hexyl isopropyl ether & 144 & ether \\
\hline 11 & 13.33 & phenylethyl alcohol & 122 & alcohol \\
\hline 12 & 13.86 & 2,4-dimethyl phenol & 122 & phenol \\
\hline 13 & 13.98 & z3-nonen-1-ol & 142 & alcohol \\
\hline 14 & 14.14 & 4-ethyl-phenol & 122 & phenol \\
\hline 15 & 14.17 & 2,3-dimethyl phenol & 122 & phenol \\
\hline 16 & 14.62 & 1-dodecene & 168 & olefin \\
\hline 17 & 14.63 & 1-methylene-1h-indene & 128 & olefin \\
\hline 18 & 14.76 & dodecane & 170 & olefin \\
\hline 19 & 15.01 & 2,3-dihydro benzofuran & 120 & furan \\
\hline 20 & 15.17 & 2-(1-methylethyl) phenol & 136 & phenol \\
\hline 21 & 15.22 & an ether & $?$ & ether \\
\hline 22 & 15.27 & 2-(2-propenyl)-1,3-dioxolane & 114 & alkane \\
\hline 23 & 15.36 & 2-ethyl-5-methyl phenol & 136 & phenol \\
\hline 24 & 15.61 & an ether & $?$ & ether \\
\hline 25 & 15.71 & an ether & $?$ & ether \\
\hline 26 & 15.78 & nonanoic acid & 158 & satured acid \\
\hline 27 & 15.92 & an ether & $?$ & ether \\
\hline 28 & 16.06 & 4-ethyl-2-methoxy phenol & 152 & phenol \\
\hline 29 & 16.25 & an ether & $?$ & ether \\
\hline 30 & 16.41 & indole & 117 & indole \\
\hline 31 & 16.48 & tridecane & 184 & alkane \\
\hline 32 & 16.62 & 2,5-diethyl phenol & 150 & phenol \\
\hline 33 & 16.73 & 2-methoxy-4-vinyl phenol & 150 & phenol \\
\hline 34 & 16.77 & unknown & $?$ & \\
\hline 35 & 17.04 & 3-(1-methylbutoxy)-2-butanol & 160 & alcohol \\
\hline 36 & 17.14 & p-mentha-1(7),8(10)-dien-9-ol & 152 & alcohol \\
\hline 38 & 17.46 & 4-hydroxy benzaldehyde & 122 & aldehyde \\
\hline 40 & 17.65 & 9-hydroxy-2-nonanone & 158 & ketone \\
\hline 41 & 17.70 & 4-propyl phenol & 136 & phenol \\
\hline 42 & 18.14 & 2-n-butyladamantane & 192 & alkane \\
\hline 43 & 18.28 & tetradecane & 198 & alkane \\
\hline 44 & 18.62 & 2-cyclohexyl-1-(1-methyl-1h-imidazol-4-yl) ethanone & 206 & ketone \\
\hline 45 & 18.91 & 1-(3-hydroxyphenyl) ethanone & 136 & ketone \\
\hline 46 & 18.93 & 1-(2-hydroxyphenyl) ethanone & 136 & ketone \\
\hline 47 & 19.20 & unknown & $?$ & \\
\hline 48 & 19.25 & a benzothiazolecarboxaldehyde & 163 & aldehyde \\
\hline 49 & 19.29 & a benzothiazolecarboxaldehyde & 163 & aldehyde \\
\hline 50 & 19.37 & ethyl 4-hydroxy-dl-mandelate & 196 & fatty acid ester \\
\hline 51 & 19.86 & 1-(4-hydroxy-3-methoxyphenyl) ethanone & 166 & ketone \\
\hline 86 & 27.92 & unknown & $?$ & \\
\hline 88 & 28.40 & isopropyl palmitate & 298 & fatty acid ester \\
\hline 91 & 29.58 & octadecanoic acid, methyl ester & 298 & fatty acid ester \\
\hline 92 & 29.93 & octadecanoic acid & 284 & satured acid \\
\hline 93 & 30.13 & hexadecanamide & 255 & amide \\
\hline 94 & 30.38 & docosane & 310 & alkane \\
\hline 95 & 31.36 & tricosane & 324 & alkane \\
\hline 96 & 31.92 & Z9-octadecenamide & 281 & amide \\
\hline
\end{tabular}

a) Compounds are numbered in the order of increasing GC retention time. Trace components are not listed. "?", Represents the compound that could not be identified. 
Table 4 Spearman's correlation coefficients $\left(R_{s}\right)$ for all sex-age groups tested according to different seasons

\begin{tabular}{|c|c|c|c|c|}
\hline & \multicolumn{2}{|c|}{ Mating season } & \multicolumn{2}{|c|}{ Non-mating season } \\
\hline & AGS & urine & AGS & urine \\
\hline Adult males & $0.262(0.464,10)$ & $-0.851(0.000,15)$ & - & $-0.134(0.242,78)$ \\
\hline Adult females & $0.000^{\mathrm{a})}(15)$ & $0.222(0.334,21)$ & - & $-0.049(0.750,45)$ \\
\hline Sub-adult males & $-0.258(0.353,15)$ & - & - & $0.200(0.475,15)$ \\
\hline Sub-adult females & $-0.149(0.518,21)$ & - & - & - \\
\hline All individuals & $0.056(0.355,276)$ & $-0.175(0.126,78)$ & - & $0.019(0.706,406)$ \\
\hline
\end{tabular}

Numbers in parentheses indicate significant values and sample size, respectively. "-”, Sample not available for analysis. a) No correlation was calculated due to their unrelated genetic relationships.

choose. Because of the large cost associated with female pandas (see Introduction), selection should be stronger on females than on males in choosing mates with appropriate levels of genetic relatedness for optimal breeding. Males, on the other hand, would attract more females if they could provide information about their genetic makeup (compatibility, quality, or identity) to be assessed by females. Hence, information about kinship should be selected to be advertised together with that about sex, age, and reproductive status ${ }^{[24,36,38]}$, as part of the Gestalt information about male attractiveness.

This leads to a next question: Why did urine from adult males, not sub-adult males, contain kinship information? Because sub-adults do not participate in reproduction, they are not selected to attract mates by providing information about kinship before maturation. Additionally, late initiation of kinship odor may also protect young males from the aggression of adult males in mate competition and thus reduce the risk of being injured $^{[28,39]}$. Sub-adult males is really present in the leklike site in the mating season, yet they are not involved in the fighting but simply looking on ${ }^{[40]}$. In terms of odor ontogeny, some of the relative abundances of fatty acids were found to be significantly and positively correlated with pandas' age by year while some alkanes negatively correlated with pandas' age ${ }^{[36]}$. They gradually gain them as they get mature ${ }^{[24]}$. Thus, the chemical profiles of the secretions change with developmental stage in the panda, presumably due to the fact that the genes associated with odor production are turned on as the animal enters puberty. A wealthy of literatures shows that luteinizing hormones ( $\mathrm{LH})$, follicle-stimulating hormone (FSH) and prolactin (PRL) from the anterior pituitary as well as androgen are correlated with increases in the weight of testes and accessory sex organs resulting from animals' growth (see review by Asa ${ }^{[41]}$ ). A subsequent study in white rhinoceros (Ceratotherium simum simum) further shows that androgen metabolite concentrations increase with sexual maturity ${ }^{[42]}$.

Along the same line, we can answer the third question: Why did kinship information show up only in the mating season? If kinship information is used by males to attract females for mating, males should produce kinship odor during at least the mating season. Its absence in the non-mating season may be due to the reason that panda kinship odor is controlled by or coupled with hormones produced or elevated only in the mating season. Indeed, many types of pheromones are produced only in this period in many other mammals. For example, territorial white rhinoceros show typical seasonal fluctuations in androgen metabolite concentrations, which in turn influence their pheromones ${ }^{[42]}$. Male specific pheromones are also found to be testosterone-dependent and known to evoke behavioral responses in conspecifics ${ }^{[33-45]}$. Moreover, hormones affect mammalian skin glands ${ }^{[46]}$. This has been well demonstrated with male goats, in which the primer pheromone is testosterone-dependent in the sebaceous gland ${ }^{[47]}$. For pandas, if the production of kinship odor depends on male hormones in the mating season, females, of course, will not produce such a pheromone. This nicely explains why adult males failed to show discrimination on female urine in a previous study by Swaisgood et al. ${ }^{[25]}$.

In the beaver, AGS contains information about genetic relatedness ${ }^{[9]}$. It is surprising that such information was missing in panda AGS, despite our effort for searching for it. Nonetheless, if males produce kinship odors as part of the advertisement "package" for females, they would not need a long-lasting effect. Urine is clearly a better place than AGS to host the kinship information for a quick effect because AGS production is slow. If this conjecture is correct, we can predict that 
males should present urinary secretion, not AGS, to females during the mating season. Behavioral observation shows that sexually active males scent-mark more frequently with urine than those of sexually inactive ones $^{[21]}$. AGS, on the contrary, has been documented to be used in competition in especially males ${ }^{[48]}$. Clearly, AGS and urine are different in their communicative functions. Like in mice, volatile compounds in urine are more susceptive to genetic variation than in preputial gland, and even a gene difference can differentiate urine volatiles $^{[49]}$.

Despite the surprising finding of the existence of an age-, sex-, and season-dependent kinship odor in the panda, we are aware of the caveats in our study due especially to some missing data for AGS in the non-mating season. This caveat, however, addresses further study on the kinship odor in the AGS in the non-mating season. The potential kinship odor exploring in the AGS in the non-mating season will be greatly helpful for revealing the mechanisms of pandas' dispersal and habitat selection. Therefore, we will have to find a new, non-invasive sampling method to circumvent the strict regulations for obtaining more complete data to corroborate our tentative findings reported here in the future. Also, we are aware that the male kinship odors uncovered in this study, despite some indirect behavioral evidence, needs

1 MacDonald D W, Brown R E. Introduction: The pheromone concept in mammalian chemical communication. In: Brown R E, MacDonald D W, eds. Social Odours in Mammals. New York: Oxford University Press, 1985. 1- 18

2 Sun L, Müller-Schwarze D. Chemical signals in the beaver: One species, two secretions, many functions? In: Johnston R E, Müller-Schwarze D, Sorensen P, eds. Advances in Chemical Signals in Vertebrates. New York: Kluwer Academic/Plenum Publishers, 1999. 281-288

3 Johnston R E, Derzie A, Chiang G, et al. Individual scent signatures in golden hamsters: Evidence for specialization of function. Anim Behav, 1993, 45(6): 1061- 1070

4 MacDonald D W. The Carnivores: Order Carnivora. In: Brown R E, Macdonald D W, eds. Social Odours in Mammals. Oxford: Oxford University Press, 1985. 619-722

5 MacDonald D W. Patterns of scent marking with urine and faeces amongst carnivore communities. Symp Zool Soc Lond, 1980, 45: $107-139$

6 Müller-Schwarze D. Chemical Ecology of Vertebrates. New York: Cambridge University Press, 2006. 536

7 Yamazaki K, Beauchamp G K, Singer A, et al. Odortypes: Their origin and composition. Proc Natl Acad Sci USA, 1999, 96(4): 1522-1525

8 Todrank J, Heth G, Johnston R E. Kin recognition in golden hamsters: Evidence for kinship odours. Anim Behav, 1998, 55(2): 377-386 to be confirmed by direct behavioral assays on female mate choice.

Uncovering the kinship odor is significant for and adds a new dimension to the practice of panda conservation. Habitat fragmentation, as it has been happening in the wild, will confine pandas to their natal places and hinder their dispersal ${ }^{[50]}$. If females do choose males based on genetic relatedness, a strong female preference for genetically appropriate males will result in a reduction in breeding as genetically suitable males become fewer due to barriers for immigration. Bounded in small, patchy habitats, females may refuse to mate with available males that are two closely related to them. The same reason may account for the severe problem in captivity where about one third of females show poor mating performance in the past ${ }^{[27]}$. Hence, strong preferences for genetically appropriate mates may lead to a slow population growth in fragmented habitats, despite food abundances in recent years for the panda. Although this hypothesis needs to be tested in the future, we have at least logically shown that chemical communication in relationship to sexual selection can potentially have a tremendous impact on panda conservation.

The authors thank Profs. LUAN Helin and YAO Wen for their help in GC-MS analysis and the staff at the China Research and Conservation Center for the Giant Panda for their assistance in sample collection.

9 Sun L, Müller-Schwarze D. Anal gland secretion codes for relatedness in the beaver, Castor canadensis. Ethology, 1998, 104(11): 917-927

10 Heth G, Todrank J, Johnston R E. Similarity in the qualities of individual odors among kin and species in Turkish (Mesocricetus brandti) and golden (Mesocricetus auratus) hamsters. J Comp Psychol, 1999, 113(3): $321-326$

11 Heth G, Todrank J, Burda H. Individual odor similarities within colonies and across species of Cryptomys mole rats. J Mammal, 2002, 83(2): $569-575$

12 Mateo J M. Kin-recognition abilities and nepotism as a function of sociality. P Roy Soc Lond B-Biol Sci, 2002, 269(1492): 721 - 727

13 Heth G, Todrank J, Busquet N, et al. Genetic relatedness assessment through individual odour similarities in mice. Biol J Linn Soc, 2003, 78(4): 595-603

14 Heth G, Todrank J, Busquet N, et al. Odour-genes covariance and differential investigation of individual odours in the Mus species complex. Biol J Linn Soc, 2001, 73(2): 213-220

15 Todrank J, Heth G. Odor-genes covariance and genetic relatedness assessments: Rethinking odor-based "recognition" mechanisms in rodents. Adv Study Behav, 2003, 32: 77-130

16 Holmes W G, Sherman P W. The ontogeny of kin recognition in two species of ground squirrels. Am Zool, 1982, 22(3): 491-517

17 Fletcher D J, Michener C D. Kin Recognition in Animals. Chichester: 
John Wiley \& Sons, 1987. 465

18 Hepper P G. Kin Recognition. Cambridge: Cambridge University Press, 1989. 457

19 Schaller G B, Hu J, Pan W, et al. The Giant Pandas of Wolong. Chicago: University of Chicago Press, 1985. 298

20 Kleiman D G. Ethology and reproduction of captive giant pandas (Ailuropoda melanoleuca). Z Tierpsychol, 1983, 62(11): 1-46

21 Liu D, Fang J, Sun R, et al. Behavioral comparison in individuals of different sexual ability in giant panda (Ailuropoda melanoleuca). Acta Zool Sin (in Chinese), 1998, 44(1): 27-34

22 Liu D, Wang L W, Zhang X T, et al. Urinary volatile profiles of the giant pandas pertaining to gender, season and sexual ability. J Beijing Normal Univ (Natl Sci), 2003, 39(1): 123-130

23 Swaisgood R R, Lindburg D G, Owen M A. The effects of sex, reproductive condition and context on discrimination of conspecific odours by giant pandas. Anim Behav, 2000, 60(2): 227-237

24 Yuan H, Liu D, Sun L, et al. Anogenital gland secretions code for sex and age in the giant panda, Ailuropoda melanoleuca. Can J Zool, 2004 82(10): $1596-1604$

25 Swaisgood R R, Lindburg D G, Zhou X. Giant pandas discriminate individual differences in conspecific scent. Anim Behav, 1999, 57(5): $1045-1053$

26 Zhang J-X, Liu D, Sun L, et al. Potential chemosignals in the anogenital gland secretion of giant pandas, Ailuropoda melanoleuca, associated with sex and individual identity. J Chem Ecol, 2008, 34(3): $398-407$

27 Zhang H M, Zhang K W, Wei R P, et al. Studies on the reproduction of captive giant pandas and artificial den at the Wolong Nature Reserve. In: Zhang A J, He G X, eds. Minutes of the International Symposium on the Protection of the Giant Panda (in Chinese). Chengdu: Sichuan Publishing House of Science \& Technology, 1994. 221-225

28 Yong Y G, Wei F W, Ye X P, et al. Mating behaviors of wild giant pandas in Foping Natural Reserve. Acta Theriol Sin (in Chinese), 2004, 24(4): 346-349

29 Anderssen M. Sexual Selection. Princeton: Princeton University Press, 1994. 599

30 Dugatkin L A, Godin J G J. How females choose their mates. Sci Am, 1998, 278(4): 56-61

$31 \mathrm{Hu}$ J. Studies on reproductive ecology of the giant panda. In: Hu J, Wei F, Yuan C, Wu Y, eds. Research and Progress in Biology of the Giant Panda (in Chinese). Chengdu: Sichuan Publishing House of Science \& Technology, 1990. 309-315

32 Wei F, Hu J, Xu G, et al. Preliminary study on the life table and reproductive characters of the wild giant panda. In: Hu J C, Wei F W, Yuan C G, Wu Y, eds. Research and Progress in Biology of the Giant Panda (in Chinese). Chengdu: Sichuan Publishing House of Science \& Technology, 1990. 177-186

33 Brown R E, MacDonald D W. Soical Odours in Mammals. Oxford, USA: Oxford University Press, 1985. 568

34 Boyse E A, Beauchamp G K, Yamazaki K, et al. Genetic components of kin recognition in mammals. In: Hepper P G, ed. Kin Recognition. Cambridge: Cambridge University Press, 1991. 148-161
35 Wang C Q, Hu J C. Behavioral ecology of the giant panda. In: Hu J C, Wei F W, Yuan C G, Wu Y, eds. Research and Progress in Biology of the Giant Panda (in Chinese). Chengdu: Sichuan Publishing House of Science \& Technology, 1990. 263-271

36 Liu D, Yuan H, Tian H, et al. Do the anogenital gland secretions of giant panda code for their sexual ability? Chin Sci Bull, 2006, 51(16): 1986-1995

37 Xie Z, Gipps J. The 2002 International Studbook for Giant Panda Beijing: Chinese Association of Zoological Gardens, 2002

38 Mateo J M. The nature and representation of individual recognition odours in Belding's ground squirrels. Anim Behav, 2006, 71(1): $141-154$

39 Pan W, Lü Z, Zhu X, et al. A Chance for Lasting Survival (in Chinese). Beijing: Beijing University Publishing House, 2001. 235

40 Wang X Q. Preliminary observations on the behavior of the giant pandas which chase to mate. In: Hu J C, Wei F W, Yuan C G, Wu Y, eds. Research and Progress in Biology of the Giant Panda (in Chinese). Chengdu: Sichuan Publishing House of Science \& Technology, 1990. $272-274$

41 Asa C S. Reproductive Physiology. In: Kleiman D G, Allen M E, Thompson K V, et al. eds. Wild Mammals in Captivity__ Principles and Techniques. Chicago: The University of Chicago Press, 1996 $390-417$

42 Kretzschmar P, Ganslosser U, Dehnhard M. Relationship between androgens, environmental factors and reproductive behavior in male white rhinoceros (Ceratotherium simum simum). Horm Behav, 2004, 45(1): $1-9$

43 Gawienowski A M, Denicola D B, Stacewiczsapuntzakis M. Androgen dependence of a marking pheromone in rat urine. Horm Behav, 1976, 7(4): 401-405

44 Novotny M, Harvey S, Jemiolo B. Chemistry of male-dominance in the house mouse, Mus domesticus. Experientia, 1990, 46(1): 109-113

45 Monahan E J, Maxson S C. Y chromosome, urinary chemosignals, and an agonistic behavior (offense) of mice. Physiol Behav, 1998 , 64(2): $123-132$

46 Ebling F J. Hormonal control of mammalian skin glands. In Müller-Schwarze D, Mozell M M, eds. Chemical Signals in Vertebrates. New York: Plenum Press, 1977. 17-39

47 Iwata E, Wakabayashi Y, Kakuma Y, et al. Testosterone-dependent primer pheromone production in the sebaceous gland of male goat. Biol Reprod, 2000, 62(3): 806-810

48 White A M, Swaisgood R R, Zhang H. The highs and lows of chemical communication in giant pandas (Ailuropoda melanoleuca) effect of scent deposition height on signal discrimination. Behav Ecol Sociobiol, 2002, 51(6): 519-529

49 Zhang J X, Rao X P, Sun L, et al. Putative chemical signals about sex, individuality and genetic background in the preputial gland and urine of the house mouse (Mus musculus). Chem Senses, 2007, 32(3): $293-303$

50 Loucks C J, Lü Z, Dinerstein E, et al. ECOLOGY: Giant pandas in a changing landscape. Science, 2001, 294(5546): 1465 